\title{
Partial transposition as a direct link between concurrence and negativity
}

\author{
Christopher Eltschka \\ Institut für Theoretische Physik, Universität Regensburg, D-93040 Regensburg, Germany \\ Géza Tóth \\ Department of Theoretical Physics, University of the Basque Country UPV/EHU, P.O. Box 644, E-48080 Bilbao, Spain; \\ IKERBASQUE, Basque Foundation for Science, E-48013 Bilbao, Spain; \\ and Wigner Research Centre for Physics, Hungarian Academy of Sciences, P.O. Box 49, H-1525 Budapest, Hungary \\ Jens Siewert \\ Department of Physical Chemistry, University of the Basque Country UPV/EHU, P.O. Box 644, E-48080 Bilbao, Spain \\ and IKERBASQUE, Basque Foundation for Science, E-48013 Bilbao, Spain
}

(Received 27 November 2014; published 27 March 2015)

\begin{abstract}
Detection of entanglement in bipartite states is a fundamental task in quantum information. The first method to verify entanglement in mixed states was the partial-transpose criterion. Subsequently, numerous quantifiers for bipartite entanglement were introduced, among them concurrence and negativity. Surprisingly, these quantities are often treated as distinct or independent of each other. The aim of this contribution is to highlight the close relations between these concepts, to show the connections between seemingly independent results, and to present various estimates for the mixed-state concurrence within the same framework.
\end{abstract}

DOI: 10.1103/PhysRevA.91.032327

PACS number(s): 03.67.Mn, 03.65.Aa

\section{INTRODUCTION}

In quantitative entanglement theory, considerable effort has been spent in developing many different entanglement measures [1-3], while much less is known regarding the relations between these measures and, in particular, their connection to the resources they quantify. This has led to a situation in which parts of quantitative entanglement theory have the reputation of a certain arbitrariness, if not a lack of meaning. As opposed to this, we believe that-given a well-defined entanglement measure-there is a physical resource (defined through a protocol) that is quantified by this measure.

At present, there are only a few well-established links between entanglement-related resources and their quantifiers [4-10]. However, many more mathematical ways of characterizing and quantifying entanglement are known than the corresponding protocols using that entanglement. Therefore, we think it is important to investigate and reveal the relations between different concepts, i.e., their possible common origins and essential differences, in order to introduce more structure in the world of entanglement measures where it is possible.

The subject of this article exemplifies the coexistence and apparent independence of different concepts in entanglement characterization. The partial-transpose criterion [11,12] provided the first possibility to detect entanglement in arbitrary mixed states. Later, numerous tools based on the partial transpose were developed, such as decomposable entanglement witnesses [13], negativity (and logarithmic negativity) as an entanglement measure [14-17], combinations of the latter in the detection of multipartite entanglement [18,19], and others. On the other hand, the concurrence was first introduced by Bennett et al. [5] as an auxiliary tool to compute the entanglement of formation for Bell-diagonal two-qubit states and then developed further by Wootters and co-workers [20-22] who established concurrence as an entanglement measure in its own right. Subsequently, generalizations to the higher-dimensional case $d \times d(d>2)$ as well as for multipartite systems (e.g., Refs. [23-30]) were proposed.

There have been comparative studies of concurrence and negativity (e.g., Refs. [15,31-35]); however, as far as we can see, they continue to exist in separate research lines. Therefore, we find it useful to present a discussion showing that both negativity and concurrence can be directly related to the partial transpose, and that both essentially determine the same type of entanglement deriving from the Schmidt rank of a Bell state, which results in various mathematical relations linking all of these quantities.

Our paper is organized as follows. In Sec. II, we introduce the most important concepts and notation. In Sec. III, we discuss the links between partial transpose, concurrence, and negativity for pure states. Finally, in Sec. IV, we extend this discussion to mixed states. In particular, we study a family of symmetric bipartite mixed states - the axisymmetric states-for which the quantitative concepts of interest can be derived exactly, thus providing both an illuminating illustration as well as a powerful tool for further investigation.

\section{DEFINITIONS}

Throughout this article, we study bipartite quantum systems with $d$-dimensional local parties (often termed $d \times d$ systems). For their pure states, $\psi \in \mathcal{H}=\mathcal{H}_{A} \otimes \mathcal{H}_{B}=\mathbb{C}^{d} \otimes \mathbb{C}^{d}$. Given orthonormal bases $\left\{|j\rangle_{A}\right\},\left\{|k\rangle_{B}\right\}$ for the two parties, a state $\psi$ can be written as

$$
|\psi\rangle=\sum_{j, k=1}^{d} \psi_{j k}|j\rangle_{A} \otimes|k\rangle_{B} \equiv \sum_{j, k=1}^{d} \psi_{j k}|j k\rangle .
$$

The mixed states $\rho$ are bounded positive operators acting on $\mathcal{H}$ and can be represented as convex combinations of pure-state 
projectors $\pi_{\psi} \equiv|\psi\rangle\langle\psi|$ (with $\operatorname{tr} \pi_{\psi}=1$ ),

$$
\rho=\sum_{j} p_{j} \pi_{\psi}
$$

where $p_{j} \geqq 0, \quad \sum_{j} p_{j}=1$. Importantly, such decomposition of a mixed state is not unique, that is, there are infinitely many pure-state ensembles representing a given state [36].

For each pure state $\psi$, there is a so-called Schmidt decomposition (see, e.g., Ref. [37]),

$$
|\psi\rangle=\sum_{j=1}^{r(\psi)} \sqrt{\lambda_{j}}\left|\alpha_{j} \beta_{j}\right\rangle
$$

with $\lambda_{j} \geqq 0$ and $r(\psi) \leqq d$. The local Schmidt bases $\left\{\left|\alpha_{j}\right\rangle_{A}\right\}$, $\left\{\left|\beta_{j}\right\rangle_{B}\right\}$ can be obtained by suitable local unitary transformations from given local bases $\left\{|k\rangle_{A}\right\},\left\{|l\rangle_{B}\right\}$. The Schmidt rank $r(\psi)$ is the number of nonvanishing Schmidt coefficients $\lambda_{j}$.

For mixed states, the generalization of the Schmidt rank is the Schmidt number [38], defined as the smallest maximum Schmidt rank occurring in any decomposition $\left\{\left(p_{j}, \psi_{j}\right)\right\}$ of $\rho$,

$$
r(\rho)=\min _{\left\{\left(p_{j}, \psi_{j}\right)\right\}} \max _{j} r\left(\psi_{j}\right)
$$

Given a state $\rho$, we may trace out one of the parties and obtain the reduced state of the other party $\rho_{A} \equiv \operatorname{tr}_{B} \rho$ (and analogously for $\rho_{B}$ ). A well-known quantifier for the entanglement of the pure state $\psi$ is the concurrence

$$
C(\psi)=\sqrt{2\left(1-\operatorname{tr} \rho_{A}^{2}\right)}=\sqrt{2\left(1-\operatorname{tr} \rho_{B}^{2}\right)} .
$$

Note that sometimes the factor 2 is replaced by $d /(d-1)$; however, this difference in normalization is not essential. Interestingly, it was shown $[24,25]$ that

$$
\begin{aligned}
C(\psi) & =\sqrt{\sum_{j k l m}\left|\psi_{j m} \psi_{l k}-\psi_{j k} \psi_{l m}\right|^{2}} \\
& =\sqrt{4 \sum_{j<l, k<m}\left|\psi_{j m} \psi_{l k}-\psi_{j k} \psi_{l m}\right|^{2}},
\end{aligned}
$$

where $c_{j k l m} \equiv \psi_{j k} \psi_{l m}-\psi_{j m} \psi_{l k}$ are the concurrence vector components [25,31].

For mixed states $\rho$, the concurrence is given by the minimum average concurrence taken over all decompositions of $\rho$, the so-called convex roof [39],

$$
C(\rho)=\min _{\left\{\left(p_{j}, \psi_{j}\right)\right\}} \sum_{j} p_{j} C\left(\psi_{j}\right) .
$$

The convex roof is notoriously hard to evaluate, therefore it is difficult to determine whether or not an arbitrary state is entangled.

The partial transpose of a bipartite state $\rho$ (with respect to party $B$ ) is given by

$$
\rho^{T_{B}}=\left[\sum_{j k l m} \rho_{j k, l m}|j k\rangle\langle l m|\right]^{T_{B}} \equiv \sum_{j k l m} \rho_{j k, l m}|j m\rangle\langle l k| .
$$

The partial transpose indicates entanglement of $\rho$ if $\rho^{T_{B}}$ has negative eigenvalues. The corresponding quantifier is the negativity [14-17],

$$
\mathcal{N}(\rho)=\frac{1}{2}\left(\left\|\rho^{T_{B}}\right\|_{1}-1\right),
$$

where $\|M\|_{1} \equiv \operatorname{tr} \sqrt{M^{\dagger} M}$ is the trace norm of the matrix $M$. The huge advantage of the negativity is that it can easily be computed for any mixed state, however, at the price that entanglement in states with a positive partial transpose (PPT) is not detected.

A related quantity which does detect PPT entanglement but, again, is hard to compute is the convex-roof extended negativity (CREN) [40,41],

$$
\mathcal{N}^{\mathrm{CREN}}(\rho)=\min _{\left\{\left(p_{j}, \psi_{j}\right)\right\}} \sum_{j} p_{j} \mathcal{N}\left(\psi_{j}\right) .
$$

It is the largest convex function that coincides with $\mathcal{N}(\psi)$ on the pure states, that is, $\mathcal{N}^{\mathrm{CREN}}(\rho) \geqq \mathcal{N}(\rho)$.

An important property of concurrence and negativity is that they are both invariant under local unitary transformations. The Schmidt rank does not change under arbitrary invertible local operations.

\section{PURE STATES}

The relation between pure-state concurrence and the partial transpose was studied before (e.g., [31,35]), but we find it important to make it very explicit here because this will directly connect to the negativity and show what is actually quantified by these measures. Moreover, we discuss how concurrence and negativity can be viewed as $\ell_{p}$ norms of the concurrence vector.

\section{A. Partial transpose and concurrence}

Assume the pure state $\phi$ is a tensor product,

$$
\begin{aligned}
|\phi\rangle & =\sum_{j k} \phi_{j k}|j k\rangle \\
& =|a\rangle \otimes|b\rangle=\sum_{j k} a_{j} b_{k}|j k\rangle,
\end{aligned}
$$

so that the corresponding projector can be written as

$$
\begin{aligned}
\pi_{\phi} & =\sum_{j k l m} \phi_{j k} \phi_{l m}^{*}|j k\rangle\langle l m| \\
& =\sum_{j k l m} a_{j} b_{k} a_{l}^{*} b_{m}^{*}|j k\rangle\langle l m| .
\end{aligned}
$$

The matrix elements of the partial transpose of $\pi_{\phi}$ instead read

$$
\left(\pi_{\phi}^{T_{B}}\right)_{j k, l m}=\phi_{j m} \phi_{l k}^{*}=a_{j} b_{k}^{*} a_{l}^{*} b_{m}
$$

Therefore, for any product state $\phi$, we have

$$
\begin{aligned}
& \left|\left(\pi_{\phi}^{T_{B}}\right)_{j k, l m}\right|^{2}-\left(\pi_{\phi}^{T_{B}}\right)_{j k, j k}\left(\pi_{\phi}^{T_{B}}\right)_{l m, l m} \\
& \quad=\left|\phi_{j m} \phi_{l k}\right|^{2}-\left|\phi_{j k} \phi_{k m}\right|^{2}=\left|\phi_{j m} \phi_{l k}-\phi_{j k} \phi_{l m}\right|^{2}=0 .
\end{aligned}
$$

A state that does not fulfill condition (14) cannot be a product state. Since violation of Eq. (14) may occur for any combination of level pairs $(j, l)$ for party $A$ and $(k, m)$ for $B$, 
we define

$$
\tilde{C}(\psi)^{2} \equiv \sum_{j k l m}\left|\psi_{j m} \psi_{l k}-\psi_{j k} \psi_{l m}\right|^{2}
$$

as a quantifier for the violation of the product-state condition for the state $\psi$. By comparing Eqs. (6) and (15), we see that $\tilde{C}(\psi)$ coincides with the $d \times d$ concurrence $C(\psi)$. That is, for a pure state $\psi$, the squared concurrence is simply a measure for the total violation of the PPT-type condition (14).

Because of the local unitary invariance of $C(\psi)$ (see Sec. IIIC), it suffices to compute the concurrence for the Schmidt decomposition $|\psi\rangle=\sum \sqrt{\lambda_{j}}\left|\alpha_{j} \beta_{j}\right\rangle$ of $\psi$, and one obtains

$$
\begin{aligned}
C(\psi) & =\sqrt{2 \sum_{j k}\left|\sqrt{\lambda_{j} \lambda_{k}} \delta_{j k}-\sqrt{\lambda_{j} \lambda_{k}}\right|^{2}} \\
& =2 \sqrt{\sum_{j<k} \lambda_{j} \lambda_{k}} .
\end{aligned}
$$

That is, the concurrence is identical to the case $k=2$ of the concurrence monotones $C_{k}$ defined by Gour [42], i.e., the $k$ th elementary symmetric function of the Schmidt coefficients taken to a power so that it is homogeneous of degree 2 in the state coefficients (note the different normalization of $C_{2}(\psi)$ in Ref. [42]).

Consider now the (maximally entangled) Bell state of rank $r$,

$$
\left|\Phi_{r}\right\rangle=\frac{1}{\sqrt{r}} \sum_{j=1}^{r}|j j\rangle .
$$

The corresponding concurrence equals

$$
C\left(\Phi_{r}\right)=\sqrt{\frac{2(r-1)}{r}} .
$$

We see that the concurrence grows monotonically with the Schmidt rank of $\Phi_{r}$. For rank-r states which are not maximally entangled, the concurrence clearly is smaller than $C\left(\Phi_{r}\right)$; in a sense, it attributes an "effective rank" $r_{\mathrm{eff}}=\frac{1}{1-\frac{1}{2} C^{2}}<r$ to the state.

\section{B. Negativity}

Again, because of the local unitary invariance, we can compute the negativity $\mathcal{N}(\psi)$ from the Schmidt decomposition $|\psi\rangle=\sum \sqrt{\lambda_{j}}\left|\alpha_{j} \beta_{j}\right\rangle$

$$
\mathcal{N}(\psi)=\sum_{j<k} \sqrt{\lambda_{j} \lambda_{k}} .
$$

In particular, for the Bell states $\Phi_{r}$, we find

$$
\mathcal{N}\left(\Phi_{r}\right)=\frac{1}{2}\left[2 \frac{r(r-1)}{2} \frac{1}{r}\right]=\frac{r-1}{2} .
$$

That is, in analogy with the concurrence, the negativity "counts" the Schmidt rank, if the state is maximally entangled. The word "counting" can be taken literally here due to the linear dependence of $\mathcal{N}\left(\Phi_{r}\right)$ on $r$ (cf. Ref. [43]).

Thus, we see that both concurrence and negativity quantify the Schmidt rank, albeit in a mathematically different manner.
If a state $\psi$ is not maximally entangled, both measures attribute a kind of effective rank to it, which is smaller than that of the maximally entangled state locally equivalent to $\psi$ [that is, equivalent under stochastic local operations and communication (SLOCC)].

From Eqs. (18) and (20), it is evident that the negativity gives equal weight to each Schmidt rank increment, while the concurrence favors increments at lower Schmidt ranks. It is not difficult to track down the origin of this difference by comparing the squared equations (16) and (19). The squared concurrence contains only the products of two different Schmidt coefficients, while the squared negativity has contributions also from products of up to four Schmidt coefficients.

Moreover, the negativity keeps increasing linearly with the Schmidt rank, while the concurrence converges to a finite value. This means that adding more dimensions to a state which already has high Schmidt rank practically does not augment the concurrence. This hints at the fact that concurrence and negativity, both being related to the Schmidt rank of the state, quantify qualitatively different resources: The resource quantified by the concurrence is already present to a high degree in a state with relatively low Schmidt rank, and can be improved beyond that only marginally. On the other hand, the negativity can grow without a limit on increasing the Schmidt rank, and this should also apply to the corresponding resource.

\section{Concurrence, negativity, and $\ell_{p}$ norms}

From Eq. (6) and also from the derivation of condition (14) leading to the total violation of the PPT condition, we see that the concurrence formally looks like the length of a Euclidean vector, i.e., it can be regarded as the $\ell_{2}$ norm of the concurrence vector. However, in our derivation of condition (14), it was by no means necessary to use the square of $\left|\psi_{j m} \psi_{l k}-\psi_{j k} \psi_{l m}\right|$. The last line of Eq. (14) is correct also without squaring it. Hence, we could have introduced a total violation of the PPT condition also as

$$
\tilde{\mathcal{N}}(\psi) \equiv \frac{1}{4} \sum_{j k l m}\left|\psi_{j m} \psi_{l k}-\psi_{j k} \psi_{l m}\right|
$$

Now, comparing Eq. (21) with the negativity calculated from the Schmidt decomposition, given by Eq. (19), it is tempting to conclude that the pure-state negativity actually equals the $\ell_{1}$ norm of the concurrence vector [44], i.e., $\mathcal{N}(\psi)=\tilde{\mathcal{N}}(\psi)$. Unfortunately, this is not correct in general. The reason is that the right-hand side in Eq. (21) may increase under local unitaries, and thus cannot represent an entanglement monotone.

To put it in different words, the negativity of a pure state $\psi$ equals the $\ell_{1}$ norm (21) of the concurrence vector $c_{j k l m}=$ $\left(\psi_{j k} \psi_{l m}-\psi_{j m} \psi_{l k}\right)$ if $\psi$ is given in the Schmidt basis. Then, this $\ell_{1}$ norm assumes its minimum,

$$
\mathcal{N}(\psi)=\min _{\text {local bases }} \tilde{\mathcal{N}}(\psi)
$$

while for other local bases, it is larger. Clearly, since the minimum of the $\ell_{1}$ norm (22) equals the negativity, it is an entanglement monotone; however, the $\ell_{1}$ norm written in a 
different basis is not (it is not even invariant under local unitaries).

Now we will prove that $\tilde{\mathcal{N}}(\psi)$ is minimized for $\psi$ given in the Schmidt basis (3). To this end, consider local unitaries $U$ and $V$ applied to the parties of $\psi$ written in the Schmidt basis,

$$
\tilde{\psi}_{a b}=\sum_{m n} U_{a m} V_{b n} \psi_{m n} \equiv \sum_{m n} U_{a m} V_{b n} \sqrt{\lambda_{m}} \delta_{m n},
$$

and use this in Eq. (21) to express $\tilde{\mathcal{N}}(\tilde{\psi})$ as

$$
\begin{aligned}
\tilde{\mathcal{N}}(\tilde{\psi}) & =\frac{1}{4} \sum_{a a^{\prime} b b^{\prime}}\left|\tilde{\psi}_{a b} \tilde{\psi}_{a^{\prime} b^{\prime}}-\tilde{\psi}_{a b^{\prime}} \tilde{\psi}_{a^{\prime} b}\right| \\
& =\frac{1}{4} \sum_{a a^{\prime} b b^{\prime}}\left|\sum_{m n} U_{a m} U_{a^{\prime} n}\left(V_{b m} V_{b^{\prime} n}-V_{b n} V_{b^{\prime} m}\right) \sqrt{\lambda_{m} \lambda_{n}}\right| .
\end{aligned}
$$

In order to proceed, we note that

$$
\begin{aligned}
& \sum_{a a^{\prime} b b^{\prime}}\left|\sum_{m n} U_{a m} U_{a^{\prime} n}\left(V_{b m} V_{b^{\prime} n}-V_{b n} V_{b^{\prime} m}\right) x_{m n}\right|^{2} \\
& \quad=2 \sum_{m n}\left|x_{m n}\right|^{2}
\end{aligned}
$$

which is easily seen by expanding $|y|^{2}=y y^{*}$. This relation also implies local unitary invariance of the concurrence.

By substituting $x_{m n}=\left(\sqrt{\lambda_{m} \lambda_{n}}-1\right)$ in Eq. (24) and applying the triangle inequalities $|a-b| \geqq|| a|-| b|| \geqq|a|-|b|$, we obtain

$$
\begin{aligned}
& 2 \sum_{m \neq n} \sqrt{\lambda_{m} \lambda_{n}} \\
& \leqq \\
& \quad \sum_{a a^{\prime} b b^{\prime}}\left|\sum_{m n}\right| U_{a m} U_{a^{\prime} n}\left(V_{b m} V_{b^{\prime} n}-V_{b n} V_{b^{\prime} m}\right) \sqrt{\lambda_{m} \lambda_{n}} \mid \\
& \quad \times\left|\sum_{m n}\right| U_{a m} U_{a^{\prime} n}\left(V_{b m} V_{b^{\prime} n}-V_{b n} V_{b^{\prime} m}\right) \mid
\end{aligned}
$$

The last factor on the right-hand side is $\leqq 1$, by virtue of the Cauchy-Schwarz inequality and the normalization of columns of unitary matrices. Hence, we have

$$
\mathcal{N}(\psi)=\mathcal{N}(\tilde{\psi})=\sum_{m>n} \sqrt{\lambda_{m} \lambda_{n}} \leqq \tilde{\mathcal{N}}(\tilde{\psi}),
$$

where, as defined above, $\psi$ is a state given in the Schmidt basis, while $\tilde{\psi}$ is obtained from $\psi$ by applying local unitaries.

Finally, it is easy to construct an example showing that, indeed, $\tilde{\mathcal{N}}$ can increase under local unitaries; consider, e.g., $\Phi_{3}$ and apply a Hadamard transform in the subspace $\{|1\rangle,|2\rangle\}$. This concludes the proof.

\section{Inequalities for concurrence and negativity}

By using the results of the previous section, a number of interesting inequalities connecting concurrence, negativity, and Schmidt rank can be proven.

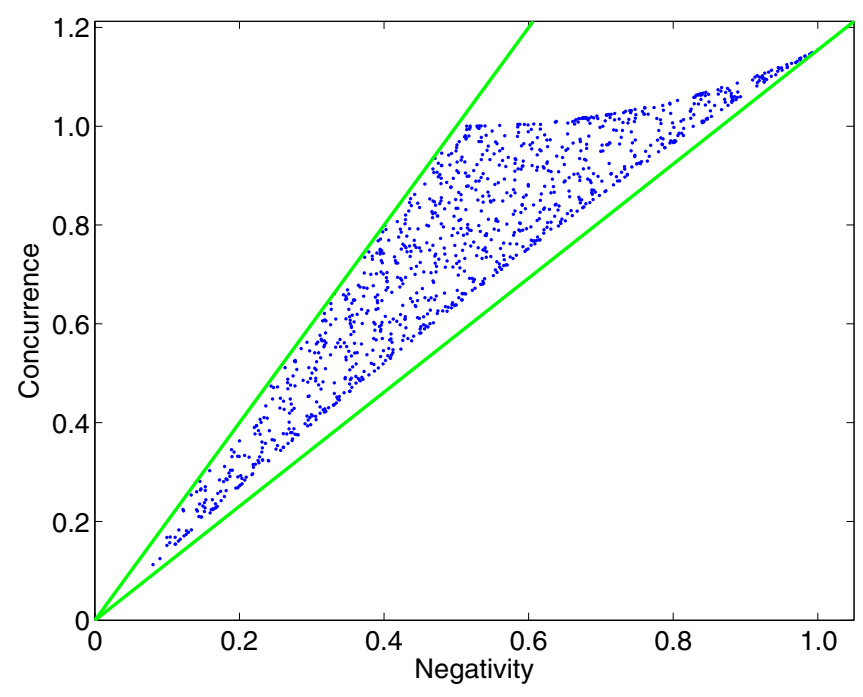

FIG. 1. (Color online) Concurrence vs negativity for 1000 random pure states [45] (blue dots) with $d=4$ and $r \leqq 3$. The green lines represent the upper and lower bounds in Eq. (27).

We have already mentioned that for a pure state $\psi \in \mathbb{C}^{d} \otimes$ $\mathbb{C}^{d}$ of Schmidt rank $r$,

$$
\begin{aligned}
C(\psi) & \leqq \sqrt{\frac{2(r-1)}{r}}, \\
\mathcal{N}(\psi) & \leqq \frac{r-1}{2} .
\end{aligned}
$$

Furthermore, we see that

$$
2 \mathcal{N}(\psi) \geqq C(\psi) \geqq 2 \sqrt{\frac{2}{r(r-1)}} \mathcal{N}(\psi) .
$$

The first of these inequalities can be readily deduced from Eqs. (16) and (19), while the second is a consequence of the fact that the quadratic is larger than the arithmetic mean. Obviously, for pure states of two qubits, the negativity equals the concurrence divided by two.

In Fig. 1, we illustrate the bounds of Eq. (27) by plotting the concurrence and negativity for many randomly chosen pure states. Clearly, those linear estimates are not the best ones possible. In fact, the evident (curved) boundaries for the concurrence values can be obtained by analytically maximizing or minimizing the concurrence for given negativity and rank of the state.

\section{MIXED STATES}

The estimation of the Schmidt number and the concurrence for arbitrary mixed states is an important problem, both for theory and experiment. By combining the results for pure states and some recent ideas $[29,43]$, one obtains an interesting toolbox for practical applications.

\section{A. Concurrence, negativity, and Schmidt number}

The great advantage of the negativity is that it can also be evaluated for arbitrary mixed states, as opposed to the 
Schmidt number or the concurrence. We will now discuss some relations connecting the negativity to the other two quantities.

It has been shown only recently [43] that the negativity can actually be used as a lower bound on the Schmidt number. Let us assume that we are given a mixed state $\rho$ of a $d \times d$ system and a decomposition $\left\{\left(p_{j}, \psi_{j}\right)\right\}$ that optimizes the Schmidt number $r(\rho)$. Then we have

$$
\mathcal{N}(\rho) \leqq \sum_{j} p_{j} \mathcal{N}\left(\psi_{j}\right) \leqq \sum_{j} p_{j} \frac{r(\rho)-1}{2} \leqq \frac{r(\rho)-1}{2}
$$

from which follows

$$
r(\rho) \geqq 2 \mathcal{N}(\rho)+1 .
$$

Here we have used Eq. (20) and the convexity of negativity. An analogous inequality can be derived for the concurrence

$$
C(\rho) \leqq \sum_{j} p_{j} C\left(\psi_{j}\right) \leqq \sqrt{\frac{2[r(\rho)-1]}{r(\rho)}},
$$

from which we arrive at

$$
r(\rho) \geqq \frac{1}{1-\frac{1}{2} C(\rho)^{2}} .
$$

This relation is essentially different from Eq. (28). On the one hand, finding a useful lower bound for the concurrence might be more difficult than calculating the negativity. On the other hand, for PPT-entangled states, Eq. (28) is not useful.

We can also find inequalities for the concurrence and the negativity. From the first inequality in Eq. (27), it follows that

$$
2 \mathcal{N}^{\mathrm{CREN}}(\rho) \geqq C(\rho),
$$

while from the second inequality of Eq. (27), for an optimal decomposition $\left\{\left(p_{j}, \psi_{j}\right)\right\}$ of the concurrence (cf. [31,35]), we get

$$
\begin{aligned}
C(\rho)=\sum_{j} p_{j} C\left(\psi_{j}\right) & \geqq 2 \sum_{j} p_{j} \sqrt{\frac{2}{r_{j}\left(r_{j}-1\right)}} \mathcal{N}\left(\psi_{j}\right) \\
& \geqq 2 \sqrt{\frac{2}{r(r-1)}} \mathcal{N}^{\mathrm{CREN}}(\rho) \\
& \geqq 2 \sqrt{\frac{2}{r(r-1)}} \mathcal{N}(\rho),
\end{aligned}
$$

where $r_{j}=r\left(\psi_{j}\right)$ and $r=\max r_{j}$. If $r$ is not known, it can be replaced by the dimension $d$.

We mention that the two-qubit concurrence divided by two equals the convex-roof extended negativity $\mathcal{N}^{\text {CREN }}$ [cf. Eq. (27)], and the negativity $\mathcal{N}$ is a lower bound to the latter. Therefore, the $2 \times 2$ negativity never exceeds the concurrence divided by two, as noted in Ref. [31]. In contrast, for higher local dimension $d>2$, the negativity may be larger than half the concurrence (e.g., Bell states), but it may also be smaller (e.g., PPT-entangled states).

\section{B. Systematic lower bounds for concurrence}

Already from the discussion in the preceding section, it can be seen that it is desirable to have systematic ways for estimating the mixed-state concurrence. However, this has proven difficult over the years. Only recently, an elegant method was devised by Huber and co-workers (based on earlier ideas $[46,47])$ to lower bound concurrence-type entanglement measures; see, e.g., Refs. [29,30]. While in those references the focus is on multipartite states, we apply it here in the simpler case of bipartite states.

The method proceeds in two steps. First, we estimate the pure-state concurrence directly from Eq. (6). Subsequently, we show that the resulting inequality applies to mixed states. We select a set $\mathcal{M}$ of $\mu$ pairs $\{j k, \operatorname{lm}\}(j<l, k<m)$ and estimate the corresponding terms in Eq. (6) using the triangle inequality and the inequality between the arithmetic and quadratic mean,

$$
\begin{aligned}
C(\psi) & \geqq \frac{2}{\sqrt{\mu}} \sum_{j k l m \in \mathcal{M}}\left|\psi_{j k} \psi_{l m}-\psi_{j m} \psi_{l k}\right| \\
& \geqq \frac{2}{\sqrt{\mu}} \sum_{j k l m \in \mathcal{M}}\left|\psi_{j k} \psi_{l m}\right|-\sqrt{\left|\psi_{j m}\right|^{2}\left|\psi_{l k}\right|^{2}} .
\end{aligned}
$$

The convex-roof construction for the concurrence and the convexity of the functions on the right-hand side of Eq. (33) guarantee that we can replace all state components by the corresponding density matrix elements so that

$$
C(\rho) \geqq \frac{2}{\sqrt{\mu}} \sum_{j k l m \in \mathcal{M}}\left|\rho_{j k, l m}\right|-\sqrt{\rho_{j m, j m} \rho_{l k, l k}} .
$$

Because of the local unitary invariance of the concurrence, one can maximize this lower bound simply by changing local bases.

A nice application of this inequality results if we choose $\mathcal{M}$ such that it specifies the off-diagonal matrix elements $\rho_{j j, k k}$ $(j<k)$ of the Bell-state projector $\pi_{\Phi_{d}}$, i.e., $\mu=\frac{1}{2} d(d-1)$. For simplicity, we replace the square-root terms by $\frac{1}{2}\left(\rho_{j k, j k}+\right.$ $\left.\rho_{k j, k j}\right) \geqq \sqrt{\rho_{j k, j k} \rho_{k j, k j}}$ and obtain

$$
\begin{aligned}
C(\rho) & \geqq \sqrt{\frac{2}{d(d-1)}} \sum_{j<k}\left(\rho_{j j, k k}+\rho_{k k, j j}-\rho_{j k, j k}-\rho_{k j, k j}\right) \\
& \geqq \sqrt{\frac{2}{d(d-1)}}\left[-1+\sum_{j<k}\left(\rho_{j j, k k}+\rho_{k k, j j}\right)+\sum_{j} \rho_{j j, j j}\right] \\
& \geqq \sqrt{\frac{2 d}{d-1}}\left[\left\langle\Phi_{d}|\rho| \Phi_{d}\right\rangle-\frac{1}{d}\right] \\
& \geqq \sqrt{\frac{2 d}{d-1}} \operatorname{tr}\left(\rho\left[\left|\Phi_{d}\right\rangle\left\langle\Phi_{d}\right|-\frac{1}{d} \mathbb{1}_{d^{2}}\right]\right)
\end{aligned}
$$

which is a concurrence estimate from the optimal Schmidt number witness [48] (for Schmidt number 2).

We can even improve this bound by optimization over local unitaries. In this way, we encounter another well-known quantity, namely, the fully entangled fraction $\mathcal{F}$ [5],

$$
\mathcal{F}(\rho)=\max _{U_{A}, U_{B}}\left\langle\Phi_{d}\left|\left(U_{A} \otimes U_{B}\right) \rho\left(U_{A} \otimes U_{B}\right)^{\dagger}\right| \Phi_{d}\right\rangle,
$$

and Eq. (35a) then reads

$$
C(\rho) \geqq \max \left(0, \sqrt{\frac{2 d}{d-1}}\left[\mathcal{F}(\rho)-\frac{1}{d}\right]\right),
$$

which is a result found in Ref. [49]. 


\section{Axisymmetric states}

We conclude our survey by considering a nontrivial family of mixed states for which the quantitative concepts we have discussed can be evaluated exactly for all finite dimensions $d$. This family is called axisymmetric states [43]. In $d$ dimensions, it includes all of those states that have the same symmetries as the Bell state $\Phi_{d}$, given by Eq. (17), namely,

(i) permutation symmetry of the two qudits,

(ii) symmetry with respect to simultaneously exchanging two levels for both qudits, e.g., $|1\rangle_{A} \leftrightarrow|2\rangle_{A}$ and $|1\rangle_{B} \leftrightarrow|2\rangle_{B}$,

(iii) simultaneous (local) phase rotations of the form

$$
V\left(\varphi_{1}, \varphi_{2}, \ldots, \varphi_{n-1}\right)=e^{i \sum \varphi_{j} \mathfrak{g}_{j}} \otimes e^{-i \sum \varphi_{j} \mathfrak{g}_{j}},
$$

where $\mathfrak{g}_{j}$ are the $(d-1)$ diagonal generators of $\mathrm{SU}(d)$. Note that the period of the phase angles $\varphi_{j}$ depends on the normalization of the generators $\mathfrak{g}_{j}$. For axisymmetric states, qubit permutation symmetry is implied by the requirements (ii) and (iii).

After discussing the symmetries of axisymmetric states, we will show how to parametrize them. In any dimension $d$, the $d \times d$ axisymmetric states are parametrized by two real numbers. This can be seen as follows. The phase rotation symmetry eliminates all off-diagonal components which are not of the form $\rho_{j j, k k}$. Qudit permutation and simultaneous level exchange symmetry are possible only if all off-diagonal elements are real and equal (one parameter) and there are only two different types of diagonal elements $\left(\rho_{j k, j k}\right.$ for $j=k$ and $j \neq k$ ) which give one more parameter, due to the normalization constraint $\operatorname{tr} \rho=1$. Based on the ideas above, we have the following parametrization:

$$
\rho_{j j, j j}^{\mathrm{axi}}=\frac{1}{d^{2}}+a, \quad \rho_{j k, j k}^{\mathrm{axi}}=\frac{1}{d^{2}}-\frac{a}{d-1}(j \neq k)
$$

$(j, k=1, \ldots, d)$, and off-diagonal entries

$$
\rho_{j l, k m}^{\mathrm{axi}}= \begin{cases}b & \text { for } l=j, m=k \\ 0 & \text { otherwise. }\end{cases}
$$

Let us now determine the limits for the parameters for physical states. We are free to choose the length scales of $a$ and $b$ in such a way that in a graphical representation, the lengths are the same as in state space, and hence geometrical intuition can be directly applied to the figures. Here the length in state space $D_{\mathrm{HS}}(A, B)$ is defined via the Hilbert-Schmidt scalar product $D_{\mathrm{HS}}^{2}(A, B)=\operatorname{tr}(A-B)$ $(A-B)^{\dagger}$. The appropriate scaling factors for the coordinates $x$ and $y$ are

$$
a=y \frac{\sqrt{d-1}}{d}, \quad b=\frac{x}{\sqrt{d(d-1)}},
$$

from which we can compute the boundary of the axisymmetric states,

$$
\begin{gathered}
-\frac{1}{d \sqrt{d-1}} \leqq y \leqq \frac{\sqrt{d-1}}{d} \\
-\frac{1}{\sqrt{d(d-1)}} \leqq x \leqq \sqrt{\frac{d-1}{d}}
\end{gathered}
$$

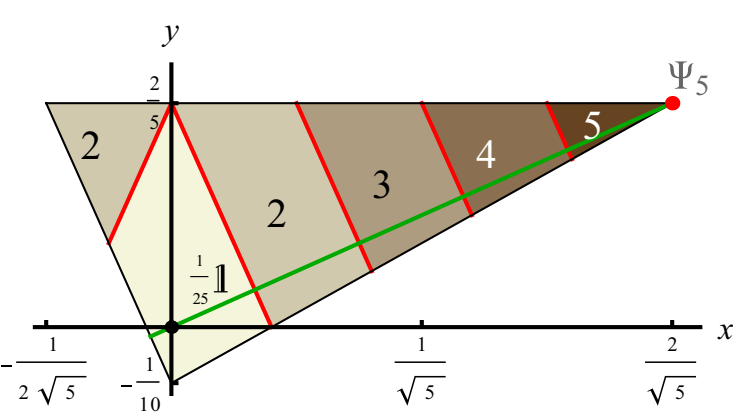

FIG. 2. (Color online) The family of $d \times d$ axisymmetric states $\rho^{\text {axi }}$ for $d=5$. It is characterized by two real parameters $x$ and $y$ describing the off-diagonal matrix elements and the asymmetry between the two types of diagonal elements, respectively [see Eqs. (39)-(41)]. The upper right corner corresponds to the $\Phi_{d}$, the only pure state in the family. The completely mixed state $\frac{1}{d^{2}} \mathbb{1}_{d^{2}}$ is located at the origin so that the isotropic states lie on the solid green line connecting the origin with the upper right corner. Note that this line is divided by the Schmidt number regions in $d$ parts of equal length. Hence the relative area of the separable states (compared to the total area of the triangle) tends to zero for $d \rightarrow \infty$ so that for axisymmetric states of large local dimension $d$, separability is the peculiar feature, rather than entanglement, in agreement with the conclusion for the entire state space in Ref. [14].

as well as

$$
-\frac{1}{\sqrt{d}}\left(y+\frac{1}{d \sqrt{d-1}}\right) \leqq x \leqq \frac{d-1}{\sqrt{d}}\left(y+\frac{1}{d \sqrt{d-1}}\right)
$$

i.e., we find a triangular shape for this family (cf. Fig. 2). In this parametrization, the completely mixed state $\frac{1}{d^{2}} \mathbb{1}_{d^{2}}$ is located at the origin, while the Bell state $\Phi_{d}$ (the only pure state in the family) appears in the upper right corner.

Based on the considerations above, we can conclude that the important isotropic states [50],

$$
\rho^{\text {iso }}=p\left|\Phi_{d}\right\rangle\left\langle\Phi_{d}\right|+\frac{1-p}{d^{2}} \mathbb{1}_{d^{2}},
$$

form a subfamily of the axisymmetric states. This is not a surprise because the symmetry group of the isotropic states is $U \otimes U^{*}$, where $U$ is an arbitrary local unitary and $U^{*}$ is its complex conjugate. The simultaneous phase rotations given by Eq. (38) form a subgroup of $U \otimes U^{*}$ (and isotropic states obey permutation and level exchange symmetry), and hence the isotropic states must be a subset of the axisymmetric family.

A particular advantage of state families defined via symmetries is that it is possible to project an arbitrary state into the families by averaging over the given symmetries [51]. Correspondingly, isotropic states can be obtained by averaging (often termed twirling) over local unitaries $U$,

$$
\mathbb{P}^{\text {iso }}(\rho)=\int d U\left(U \otimes U^{*}\right) \rho\left(U \otimes U^{*}\right)^{\dagger},
$$

while axisymmetric states arise from twirling over the operations $\mathcal{V}$ including permutations and the local unitaries $V$ in 
Eq. (38),

$$
\mathbb{P}^{\mathrm{axi}}(\rho)=\int d \mathcal{V} \mathcal{V} \rho \mathcal{V}^{\dagger}
$$

In these expressions, the integral $\int d X$ denotes the average over the corresponding symmetry group including the discrete symmetries. We mention already at this point that these averages do not increase the entanglement in the projection $\mathbb{P}^{\text {axi }}: \rho \rightarrow \rho^{\text {axi }}$ (and analogously for isotropic states) because neither permutations nor local unitaries or mixing can increase entanglement.

The procedure for performing the average in Eq. (46) in practice is easy: Given an arbitrary $d \times d$ state $\rho$, the matrix elements of its projection $\rho^{\mathrm{axi}}(\rho)$ are

$$
\begin{aligned}
\rho_{j j, j j}^{\mathrm{axi}} & =\frac{1}{d} \sum_{m} \rho_{m m, m m}, \\
\rho_{j k, j k}^{\mathrm{axi}} & =\frac{1}{d(d-1)} \sum_{m \neq n} \rho_{m n, m n}(j \neq k),
\end{aligned}
$$

with $j, k=1, \ldots, d$, and off-diagonal elements

$$
\begin{aligned}
& \rho_{j j, k k}^{\mathrm{axi}}=\frac{1}{d(d-1)} \sum_{m>n}\left(\rho_{m m, n n}+\rho_{n n, m m}\right), \\
& \rho_{j k, l m}^{\mathrm{axi}}=0 \quad \text { for } \quad k \neq j \quad \text { or } \quad l \neq m .
\end{aligned}
$$

\section{Entanglement of axisymmetric states}

The optimal Schmidt number witness [48]

$$
\mathcal{W}=\frac{k-1}{d} \mathbb{1}_{d^{2}}-\left|\Phi_{d}\right\rangle\left\langle\Phi_{d}\right|
$$

for Schmidt number $k$ can be used to detect the exact boundaries of the different SLOCC classes (for $x>0$ ), that is, the regions of different Schmidt number (see Fig. 2). While for $x<0$ the witness cannot be applied, one can check that the projection $\mathbb{P}^{\text {axi }}\left(\psi_{0}\right)$ of the product state $\left|\psi_{0}\right\rangle=\frac{1}{2}(|1\rangle+$ $|2\rangle) \otimes(|1\rangle-|2\rangle)$ is the endpoint of the border for separable states. It is also easy to verify that above the line connecting this point with the separable state at $\left(x=0, y=\sqrt{\frac{d-1}{d}}\right)$, the negativity becomes nonzero, but does not exceed 1 . The state at the upper left corner is a state of, at most, $r=2$ since it is the projection of $\frac{1}{\sqrt{2}}(|11\rangle-|22\rangle)$, and therefore the entangled states for $x<0$ must have $r=2$.

The states with Schmidt number $\leqq k$ belong to convex sets $S_{k}$ and form a hierarchy $S_{1} \subset S_{2} \subset \ldots \subset S_{d}$. Schmidt number $k=1$ corresponds to separable states (see Fig. 2). Notably, all of the boundaries are represented by straight lines. In fact, this is a hint that the bipartite case-even for large $d$-is more treatable than the multipartite case, where also for highly symmetric families of states the borders between entanglement classes are complicated (cf. [52]).

Let us now consider the entanglement quantitatively. The formula for the negativity (9) is readily applied to $\rho^{\text {axi }}$ and

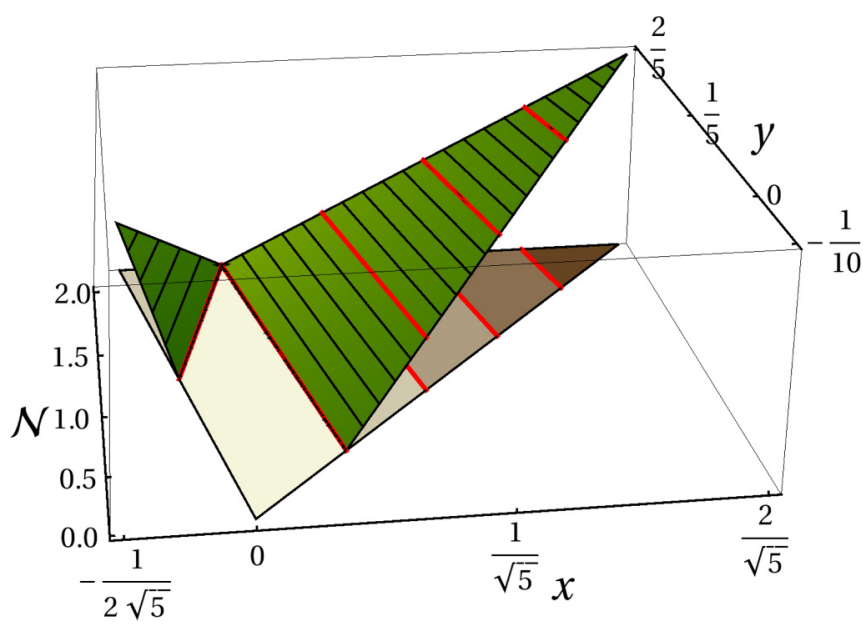

FIG. 3. (Color online) Negativity for the axisymmetric states with $d=5$ according to Eq. (50). The results for the concurrence $C\left(\rho^{\text {axi }}\right)$ are qualitatively identical; the only difference is a scaling factor $\frac{1}{2} \sqrt{\frac{2 d}{d-1}}$. The solid red lines indicate the borders between entanglement classes. Note that these are lines of constant fidelity, thus providing a nice illustration for the estimate in Eq. (37).

gives

$$
\begin{aligned}
\mathcal{N}\left(\rho^{\mathrm{axi}}(x, y)\right)= & \max \left\{0, \frac{1}{2}[\sqrt{d(d-1)}|x|\right. \\
& \left.\left.+\sqrt{d-1} y-\frac{d-1}{d}\right]\right\} .
\end{aligned}
$$

On the other hand, by using the lower bound (34) for the concurrence, we obtain

$$
\begin{aligned}
C\left(\rho^{\mathrm{axi}}(x, y)\right) \geqq & \max \left\{0, \sqrt{\frac{2}{d(d-1)}}[\sqrt{d(d-1)}|x|\right. \\
& \left.\left.+\sqrt{d-1} y-\frac{d-1}{d}\right]\right\} .
\end{aligned}
$$

These results (see Fig. 3) are remarkable for several reasons. Both concurrence and fidelity estimates depend linearly on $|x|$ and $y$ coordinates. Since Eqs. (50) and (51) include the exact values for the pure state $\Phi_{d}$, and their graphs are planes, there cannot be a larger convex function containing $\mathcal{N}\left(\Phi_{d}\right)$ and $C\left(\Phi_{d}\right)$ than these graphs. Hence, the formulas (50) and (51) represent the exact solutions for the convex-roof extended negativity and the concurrence of axisymmetric states, respectively. Moreover, it follows that all PPT axisymmetric states are separable. We mention that the concurrence result restricted to the isotropic states was found in Ref. [26].

Another immediate consequence is that the integer part of $\left[2 \mathcal{N}\left(\rho^{\mathrm{axi}}\right)+1\right]$ changes by 1 whenever a border between SLOCC classes, i.e., Schmidt numbers for $\rho^{\text {axi }}$, is crossed. That is, for axisymmetric states, Eq. (28) can be written as

$$
r\left(\rho^{\mathrm{axi}}\right)=\left\lceil 2 \mathcal{N}\left(\rho^{\mathrm{axi}}\right)\right\rceil+1,
$$

with the ceiling function $\lceil x\rceil$ denoting the smallest integer greater than or equal to $x$.

Concluding this section, we discuss yet another procedure to determine a lower bound for the convex-roof extended 
negativity and the concurrence of arbitrary $d \times d$ states $\rho$. As we have mentioned before, the symmetrization given by Eqs. (46)-(48b) does not increase the entanglement,

$$
\begin{aligned}
\mathcal{N}^{\mathrm{CREN}}\left(\mathbb{P}^{\mathrm{axi}}(\rho)\right) & \leqq \mathcal{N}^{\mathrm{CREN}}(\rho), \\
C\left(\mathbb{P}^{\mathrm{axi}}(\rho)\right) & \leqq C(\rho),
\end{aligned}
$$

so that after symmetrizing $\rho$, we can simply read off the value for $\mathcal{N}^{\mathrm{CREN}}\left(\mathbb{P}^{\text {axi }}(\rho)\right)$ or $C\left(\mathbb{P}^{\text {axi }}(\rho)\right)$ from Fig. 3. As discussed in Sec. IV B, we can maximize the state $\rho$ over local unitaries before projecting it and thus obtain an optimized lower bound.

It is interesting to note that for the concurrence, this bound coincides with the one obtained from Eq. (37). The latter can be regarded as the result of a projection of the optimized state onto the isotropic states. Thus we see that one does not lose entanglement information projecting directly onto the isotropic rather than the axisymmetric states. This is a direct consequence of the fact that essential entanglement-related information of a bipartite state is contained in its fidelity with the maximally entangled state $\Phi_{d}$, i.e., the fully entangled fraction.

\section{CONCLUSION}

We have pointed out and made explicit that not only negativity but also concurrence is closely related to the partial transposition of a $d \times d$ density matrix. In fact, both measures may be understood and derived as quantifiers for the violation of the PPT criterion in pure states. We have discussed that both negativity and concurrence quantify the Schmidt rank of a pure state, however, in different mathematical ways, which hints at the fact that they quantify different resources. Finally, we have shown that while the concurrence equals the $\ell_{2}$ norm of the concurrence vector of a pure state, the negativity is in general larger than the $\ell_{1}$ norm of the concurrence vector. The negativity equals that $\ell_{1}$ norm if the pure state is written in the Schmidt decomposition.

These relations between negativity and concurrence lead to various estimates for those measures (as well as for the Schmidt number) in mixed states. A particularly nice result is that the negativity represents a direct lower bound to the Schmidt number of a state, given by Eq. (28). In the last section, we have provided an extensive discussion of the axisymmetric states, i.e., a nontrivial family of highly symmetric $d \times d$ states for which all of the entanglement properties studied in this article can be calculated exactly.

\section{ACKNOWLEDGMENTS}

This work was funded by the German Research Foundation within SPP 1386 (C.E.), by Basque Government Grant No. IT-472-10, MINECO Grants No. FIS2012-36673-C03-01 and No. FIS2012-36673-C03-03, and UPV/EHU Program No. UFI 11/55 (J.S. and G.T.), the EU (ERC Starting Grant GEDENTQOPT, CHIST-ERA QUASAR), and National Research Fund of Hungary OTKA (Contract No. K83858). C.E. and J.S. thank M. Huber and G. Sentís for stimulating discussions, and J. Fabian and K. Richter for their support.
[1] R. Horodecki, P. Horodecki, M. Horodecki, and K. Horodecki, Rev. Mod. Phys. 81, 865 (2009).

[2] O. Gühne and G. Toth, Phys. Rep. 474, 1 (2009).

[3] C. Eltschka and J. Siewert, J. Phys. A: Math. Theor. 47, 424005 (2014).

[4] C. H. Bennett, H. J. Bernstein, S. Popescu, and B. Schumacher, Phys. Rev. A 53, 2046 (1996).

[5] C. H. Bennett, D. P. DiVincenzo, J. A. Smolin, and W. K. Wootters, Phys. Rev. A 54, 3824 (1996).

[6] M. Horodecki, P. Horodecki, and R. Horodecki, Phys. Rev. A 60, 1888 (1999).

[7] J. Grondalski, D. M. Etlinger, and D. F. V. James, Phys. Lett. A 300, 573 (2002).

[8] L. Pezzé and A. Smerzi, Phys. Rev. Lett. 102, 100401 (2009).

[9] P. Hyllus, W. Laskowski, R. Krischek, C. Schwemmer, W. Wieczorek, H. Weinfurter, L. Pezzé, and A. Smerzi, Phys. Rev. A 85, 022321 (2012).

[10] G. Tóth, Phys. Rev. A 85, 022322 (2012).

[11] A. Peres, Phys. Rev. Lett. 77, 1413 (1996).

[12] M. Horodecki, P. Horodecki, and R. Horodecki, Phys. Lett. A 223, 1 (1996).

[13] M. Lewenstein, B. Kraus, J. I. Cirac, and P. Horodecki, Phys. Rev. A 62, 052310 (2000).

[14] K. Zyczkowski, P. Horodecki, A. Sanpera, and M. Lewenstein, Phys. Rev. A 58, 883 (1998).

[15] J. Eisert and M. B. Plenio, J. Mod. Opt. 46, 145 (1999).

[16] J. Eisert, Ph.D. thesis, University of Potsdam, 2001.
[17] G. Vidal and R. F. Werner, Phys. Rev. A 65, 032314 (2002).

[18] B. Jungnitsch, T. Moroder, and O. Gühne, Phys. Rev. Lett. 106, 190502 (2011).

[19] M. Hofmann, T. Moroder, and O. Gühne, J. Phys. A: Math. Theor. 47, 155301 (2014).

[20] S. Hill and W. K. Wootters, Phys. Rev. Lett. 78, 5022 (1997).

[21] W. K. Wootters, Phys. Rev. Lett. 80, 2245 (1998).

[22] V. Coffman, J. Kundu, and W. K. Wootters, Phys. Rev. A 61, 052306 (2000).

[23] P. Rungta, V. Buzek, C. M. Caves, M. Hillery, and G. J. Milburn, Phys. Rev. A 64, 042315 (2001).

[24] S. Albeverio and S. M. Fei, J. Opt. B 3, 223 (2001).

[25] P. Badziag, P. Deuar, M. Horodecki, P. Horodecki, and R. Horodecki, J. Mod. Opt. 49, 1289 (2002).

[26] P. Rungta and C. M. Caves, Phys. Rev. A 67, 012307 (2003).

[27] S. J. Aktharshenas, J. Phys. A: Math. Gen. 38, 6777 (2005).

[28] P. J. Love, A. Maassen v. d. Brink, A. Yu. Smirnov, M. H. S. Amin, M. Grajcar et al., Quantum Inf. Proc. 6, 187 (2007).

[29] Z.-H. Ma, Z.-H. Chen, J.-L. Chen, C. Spengler, A. Gabriel, and M. Huber, Phys. Rev. A 83, 062325 (2011).

[30] J.-Y. Wu, H. Kampermann, D. Bruß, C. Klöckl, and M. Huber, Phys. Rev. A 86, 022319 (2012).

[31] F. Verstraete, K. Audenaert, J. Dehaene, and B. De Moor, J. Phys. A: Math. Gen. 34, 10327 (2001).

[32] K. Zyczkowski and I. Bengtsson, Ann. Phys. 295, 115 (2002).

[33] A. Miranowicz and A. Grudka, Phys. Rev. A 70, 032326 (2004).

[34] A. Miranowicz and A. Grudka, J. Opt. B 6, 542 (2004). 
[35] K. Chen, S. Albeverio, and S. M. Fei, Phys. Rev. Lett. 95, 040504 (2005).

[36] L. P. Hughston, R. Jozsa, and W. K. Wootters, Phys. Lett. A 183, 14 (1993).

[37] J. Preskill, Lecture notes on quantum computation (1998), http://theory.caltech.edu/ preskill/ph229/.

[38] B. M. Terhal and P. Horodecki, Phys. Rev. A 61, 040301 (2000).

[39] A. Uhlmann, Entropy 12, 1799 (2010).

[40] S. Lee, D.-P. Chi, S.-D. Oh, and J. Kim, Phys. Rev. A 68, 062304 (2003).

[41] C. Zhang, S. Yu, Q. Chen, and C. H. Oh, Phys. Rev. Lett. 111, 190501 (2013).

[42] G. Gour, Phys. Rev. A 71, 012318 (2005).

[43] C. Eltschka and J. Siewert, Phys. Rev. Lett. 111, 100503 (2013).

[44] Z.-H. Ma, Z.-H. Chen, and S.-M. Fei, Phys. Rev. A 90, 032307 (2014).

[45] The random states were obtained as follows. First, random states of a given Schmidt rank was generated in the computational basis as the Schmidt basis. Then, a random unitary rotation was applied. The algorithm provided unitaries distributed uniformly according to the Haar measure. The 1000 points were selected from the results of 100000 random states such that they uniformly fill the area on the plot. For information regarding generating random unitaries, see, for example, G. Tóth, Comput. Phys. Commun. 179, 430 (2008).

[46] O. Gühne and M. Seevinck, New J. Phys. 12, 053002 (2010).

[47] M. Huber, F. Mintert, A. Gabriel, and B. C. Hiesmayr, Phys. Rev. Lett. 104, 210501 (2010).

[48] A. Sanpera, D. Bruss, and M. Lewenstein, Phys. Rev. A 63, 050301(R) (2001).

[49] M.-J. Zhao, Z.-G. Li, S.-M. Fei, and Z.-X. Wang, J. Phys. A: Math. Theor. 43, 275203 (2010).

[50] M. Horodecki and P. Horodecki, Phys. Rev. A 59, 4206 (1999).

[51] K. G. H. Vollbrecht and R. F. Werner, Phys. Rev. A 64, 062307 (2001).

[52] C. Eltschka and J. Siewert, Phys. Rev. Lett. 108, 020502 (2012). 